\title{
Auch die geistig-seelische Verfassung beeinflusst unsere Lebenserwartung
}

\begin{abstract}
Bisher wurde in der epidemiologischen Forschung das Augenmerk überwiegend auf somatische und psychiatrische Erkrankungen gelegt, wenn man Risikofaktoren erkennen wollte, die für eine Behinderung oder erhöhte Sterblichkeit im Alter ausschlaggebend sind. Die seelische Verfassung wird meist ignoriert.
\end{abstract}

- In einer spanischen repräsentativen Längsschnittsstudie bei insgesamt 2516 Personen mit einem mittleren Alter von 75,7 Jahren wurde der Zusammenhang zwischen der geistig-seelischen Verfassung und der Sterblichkeit untersucht, unabhängig von bestehenden Erkrankungen und Behinderungen. Die subjektiven Angaben zur Zufriedenheit mit sich selbst, zum Gefühl, gebraucht zu werden und zur Akzeptanz, unabänderliche Dinge hinzunehmen, wurden mit der Philadelphia-Geriatric-Centre-Moral-Scale erfragt und bewertet.

Die Autoren fanden einen starken statistischen Zusammenhang zwischen gedrückter Stimmung und Sterblichkeit. Dieser Zusammenhang bestand

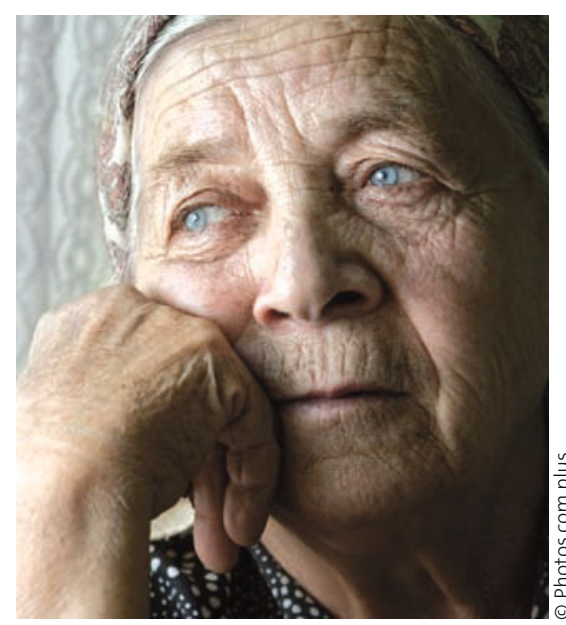

Schlechte Stimmung verkürzt das Leben.

auch noch nach Anpassung des statistischen Modells für Depression, Einnahme von Antidepressiva, sowie multiple chronische Erkrankungen und Behinderungen. Die Autoren sehen dies als Beweis an, dass eine schlechte geistigseelische Verfassung einen unabhängigen Risikofaktor für frühzeitige Sterblichkeit darstellt und bei zukünftigen Interventionsstrategien entsprechend berücksichtigt werden sollte.

\section{Kommentar}

Neben den bekannten klassischen Risikofaktoren zum Krankheitsentstehen stellt die Einstellung zu sich, seiner Gesundheit und zum Lebensumfeld einen weiteren wichtigen Baustein dar, der unsere Lebenserwartung beeinflusst. Das Erlernen von Strategien zur Bewältigung von chronischen Krankheiten und Behinderungen, das Akzeptieren von körperlichen und geistigen Grenzen mit fortschreitendem Lebensalter und die Zufriedenheit mit den eigenen Lebensumständen stellen einen weiteren Zweig für präventive Maßnahmen dar, um ein zufriedenes Altern bei möglichst langer Gesundheit zu erreichen. Die in der spanischen Untersuchung gefundenen Ergebnisse müssen jetzt noch in weiteren Kulturkreisen, z. B. im deutschen sprachraum, bestätigt werden.

T. NiKOLAUS

\footnotetext{
- J. Benito-Leon et al.

Low morale is associated with increased risk of mortality in the elderly: a population-based prospective study (NEDICES), Age and Ageing 39 (2010) 366-373
}

\section{Pelargonium sidoides bei akuter Bronchitis}

- Ein internationales Team prüfte in einer multizentrischen Studie, ob Pelargonium sidoides die Symptomatik einer akuten Bronchitis dosisabhängig lindert. 406 Patienten wurden in vier Gruppen randomisiert, die entweder 10, 30, 60, 90 mg Extrakt oder Placebo sieben Tage lang einnehmen sollten. Der Hauptzielparameter war ein validierter Symptomscore.

Die Auswertungen zeigen, dass alle drei Verumgruppen dem Placebo überlegen waren. Ein Effektoptimum schien mit der Dosis von $60 \mathrm{mg}$ pro
Tag erreicht zu sein. Die Nebenwirkungen waren mild und ebenfalls dosisabhängig. Die Autoren ziehen den Schluss, dass die Dosis von $60 \mathrm{mg} / \mathrm{Tag}$ effektiv und otpimal sei.

\section{Kommentar}

Die Studie liefert klinisch aussagekräftige Daten. Die Dosisabhängigkeit festigt den Wirkungsnachweis von Pelargonium sidoides und erlaubt eine Definition der optimalen Dosis. Die Nebenwirkungsraten sind bei dieser Dosis kaum von denen unter Placebo zu unterscheiden.
Das impliziert, dass der Extrakt unbedenklich ist, was bei der Therapie einer relative gutartigen, zeitlich limitierten Erkrankung nicht unerheblich ist. Fazit: Pelargonium sidoides ist bei der akuten Bronchitis indiziert und sollte die leider immer noch häufig verschriebenen Antibiotika ablösen.

E. ERNST =

\section{- H. Matthys et al.}

Efficacy and tolerability of EPs 7630 tablets in patients with acute bronchitis: a randomised, double-blind, placebo-controlled dose-finding study with a herbal drug preparation from Pelargonium sidoides. Curr. Med. Res. Opin. 26 (2010) 1413-1422 